\title{
Ocean Salinity Observations with SMOS Mission
}

\author{
M. Martin-Neira ${ }^{(1)}$, J. Font ${ }^{(2)}$, M. Srokosz ${ }^{(3)}$, I. Corbella $^{(4)}$, A. Camps $^{(4)}$ \\ (1) European Space Agency \\ ESTEC, Keplerlaan 1, 2200-AG Noordwijk, The Netherlands \\ Phone: +31-71-565 4052; Fax:+31-71-565 4596; E-mail: mneira@estec.esa.nl \\ (2) Instituto de Ciencias del Mar - ICM/CSIC (Spain) \\ (3) Southampton Oceanography Centre-SOC (UK) \\ (4) Polytechnic University of Catalonia - UPC (Spain) \\ E-mail: jfont@icm.csic.es; mas@soc.soton.ac.uk; corbella@tsc.upc.es; camps@voltor.upc.es
}

\section{INTRODUCTION}

The purpose of this paper is to present the capabilities of SMOS (Soil Moisture and Ocean Salinity mission) for the global mapping of ocean salinity from space. SMOS has been selected by the European Space Agency as the second Earth Explorer Opportunity with a launch date in June 2005. The sensor embarked on SMOS is MIRAS, a Microwave Imaging Radiometer with Aperture Synthesis. MIRAS works at L-band, in the two-polarisations, and has full polarimetric capability.

The measurement of sea surface salinity (SSS) is one of the challenges of SMOS. This paper presents first the scientific requirements for a number of oceanographic applications. The scientific requirements are then translated into instrument accuracy, sensitivity, stability and spatial resolution. Major sources of error in the retrieval of ocean salinity will be addressed through an experimental campaign which is described.

\section{SCIENTIFIC OBJECTIVES}

Knowledge of the distribution of salt in the global ocean and its annual and inter-annual variability, are crucial in understanding the role of the ocean in the climate system. Ocean circulation is mainly driven by the momentum and heat fluxes through the atmosphere-ocean interface, which can be traced by observation of SSS. In addition, salinity also determines ocean density and hence thermohaline circulation. In some regions (e.g. the Arctic), salinity is the most important variable as it controls processes such as deep water formation which is a key component in the ocean thermohaline circulation "conveyor belt». Ocean salinity is also linked to the oceanic carbon cycle, as it plays a part in establishing the chemical equilibrium, which in turn regulates the $\mathrm{CO}_{2}$ uptake and release. Therefore, the assimilation of SSS into global ocean biogeochemical models could improve estimates of the absorption of $\mathrm{CO}_{2}$ by the oceans.

Monitoring SSS could also be used to improve the quality of ENSO (El Niño - Southern Oscillation) prediction by numerical models. Presently the models assimilate temperature and/or altimeter-derived sea level data only. The lack of salinity measurements results in major discrepancies between modeled and observed surface currents. For example, a 0.5 psu (practical salinity units) error accounts for a $3.8 \mathrm{~cm} / \mathrm{s}$ error in geostrophic velocity at $1 \mathrm{~km}$ depth calculated from the corresponding surface value. This is particularly important in the Western Equatorial Pacific where there is a strong ENSO-related near-surface salinity signal and where zonal advection is of major importance for ENSO mechanisms.

SSS is correlated with estimates of the net evaporation minus precipitation (E-P) balance. (E-P) is difficult to measure accurately over the ocean, so global maps of SSS would provide a constraint on estimates of $(E-P)$ on the global scale. This would give insights into the phenomena driving the thermohaline circulation and also allow validating latent heat flux estimates. The water flux through the sea surface layers is critical for density stratification and strongly influences the mixed layer depth and the intensity of surface currents.

The dielectric constant for seawater is determined, among other variables, by salinity. In principle it is possible to retrieve SSS from microwave measurements as long as variables influencing the brightness temperature $\left(T_{B}\right)$ signal (SST, roughness, foam,) can be accounted for e.g. by the use of different viewing angles, polarisations and frequencies. The sensitivity of $T_{B}$ to SSS is maximum at low microwave frequencies and the good conditions for salinity retrieval are found at $\mathrm{L}$-band $(1.4 \mathrm{GHz}, 21 \mathrm{~cm}$ wavelength). However, it must be stressed that at this frequency the sensitivity of $T_{B}$ to SSS is low $(0.5 \mathrm{~K}$ per psu for an SST of $20^{\circ} \mathrm{C}$, decreasing to $0.25 \mathrm{~K}$ per psu for an SST of $0^{\circ}$ ), placing demanding requirements on the performance of the instrument (refer to Fig. 1).

Due to this low sensitivity, and the spatial resolution that can be expected with a spaceborne microwave interferometric radiometer, we are still not in a position to obtain SSS data for mesoscale or regional studies. However, several phenomena extremely relevant for large- 
scale and climatic studies can benefit from such an observation approach: barrier layer effects on tropical Pacific heat flux, halosteric adjustment of heat storage from sea level, North Atlantic thermohaline circulation, surface freshwater flux balance, etc. Considering the resolution constrains the overall goal for SSS retrieval from SMOS data is to meet the GODAE (Global Ocean Data Assimilation Experiment) optimised requirement for open ocean SSS: a resolution of $0.1 \mathrm{psu}$ over $200 \mathrm{~km}$ boxes every 10 days.

The emissitivity of the sea surface is a function of salinity, temperature and wind speed as a minor parameter. The best sensitivity to the $\mathrm{OS}$ is obtained at $1.4 \mathrm{GHz}$, however, the influence of sea temperature can not be neglected even at these frequencies. Hence, the measurement of the ocean salinity requires the use of another spacecraft, which allows the retrieval of SST.

\section{MIRAS CONFIGURATION}

The instrument for SMOS mission is MIRAS, a 2D interferometric radiometer designed to meet the main requirements of the mission [1]. It consists of a total of 81 small antenna receivers uniformly arranged on a Y-shape structure, and a total power radiometer in its center for absolute calibration. To avoid alias, the minimum antenna spacing for this configuration is $\lambda / \sqrt{3}=0.57 \lambda$, being $\lambda$ the wavelength at the central frequency. Due to the high brightness temperature contrast between the Earth and the sky, a limited amount of aliasing is permitted, as long as only affects the sky, so the selected antenna spacing is set to $0.89 \lambda$. For 27 antennas per arm, this gives a total arm length of about $5.2 \mathrm{~m}$, corresponding to an angular resolution of less than $2^{\circ}$. The instrument will be put on a low Earth sun-synchronous orbit with the antenna boresight tilted with respect to nadir, so the angular resolution translates to a ground spatial resolution better than $50 \mathrm{Km}$.

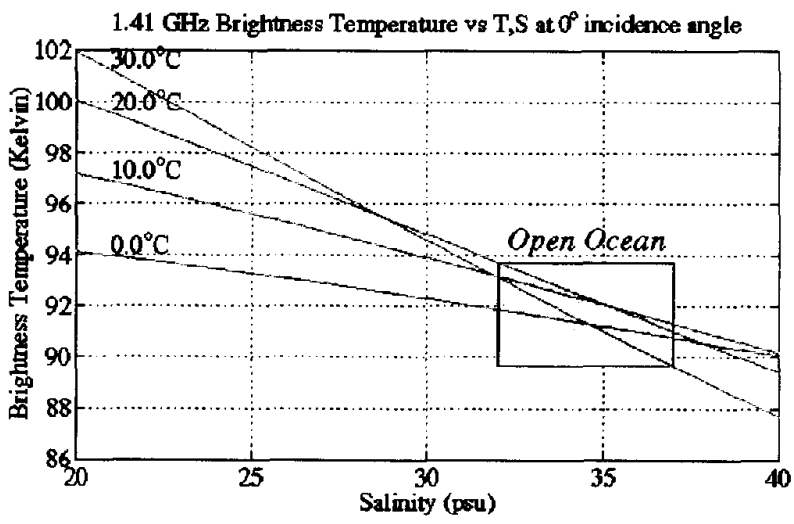

Figure 1

Brightness Temperature vs SSS for Different SST (courtesy from Gary Lagerloef)

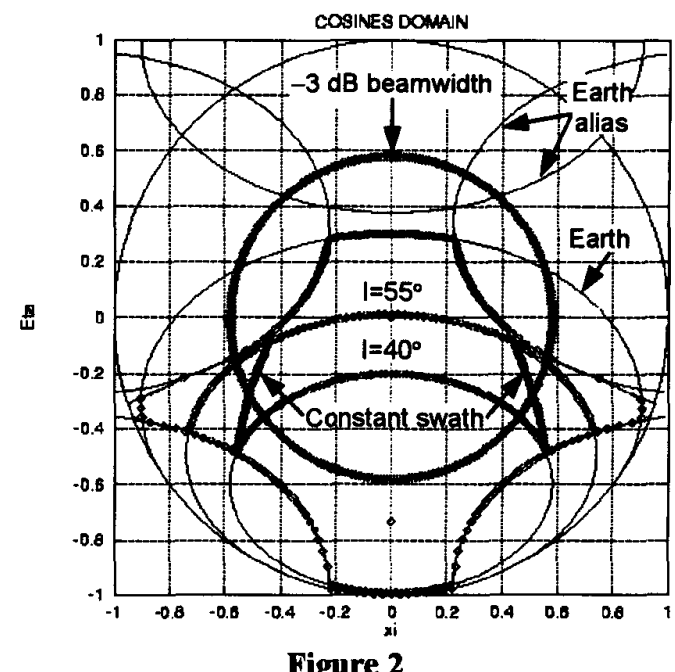

Figure 2

Typical MIRAS Field of View (courtesy CASA and GMV)

The on-ground field of view (FOV) of the instrument has a distorted hexagon shape with curved sides, having each pixel different sizes and incidence angles (Fig.2). A snap-shot brightness temperature map of this field of view is obtained each $0.78 \mathrm{~s}$, with an average resolution of about $5 \mathrm{~K}$ over $200 \mathrm{~K}$ [2]. Individual resolution of a given pixel is in fact weighted by the antenna pattern.

Due to the motion of the platform, each pixel is measured several times with different spatial and radiometric resolution and incidence angle. Provided good models exist to account for these varying measuring conditions, the salinity map can be obtained by averaging out all the retrieved values at each snap-shot. The minimum FOV along-track dimension is about $800 \mathrm{Km}$, which at 7 $\mathrm{km} / \mathrm{s}$ corresponds to $114 \mathrm{~s}$, or $146 \mathrm{snap}$-shots of $0.78 \mathrm{~s}$ each. The equivalent radiometric resolution turns out to be then $0.4 \mathrm{~K}$, corresponding to a single-pass salinity resolution of $0.8 \mathrm{psu}$ at $20^{\circ} \mathrm{C}$ or $1.6 \mathrm{psu}$ at $0^{\circ} \mathrm{C}$. However, in the center of the FOV, the total along track distance is about $1400 \mathrm{~km}$, giving 0.6 and $1.2 \mathrm{psu}$ salinity resolutions. To improve these figures, averaging of different pixels or satellite passes is needed, which can be done due to the slow timespace variation of salinity in open ocean.

The instrument will be periodically calibrated using a noise injection mechanism in combination with a highly stable noise injection radiometer. It is expected that this mechanism will provide an absolute radiometric accuracy below $2 \mathrm{~K}$.

\section{THE WISE CAMPAIGN}

The range of incidence angles of MIRAS goes from $0^{\circ}$ at nadir to approximately $60^{\circ}$, for pixels in the satellite's ground-track. This translates into a brightness temperature 
range at vertical and horizontal polarizations from 50 to 150 $\mathrm{K}$ with a small dependence on wind speed (Fig. 3 ).

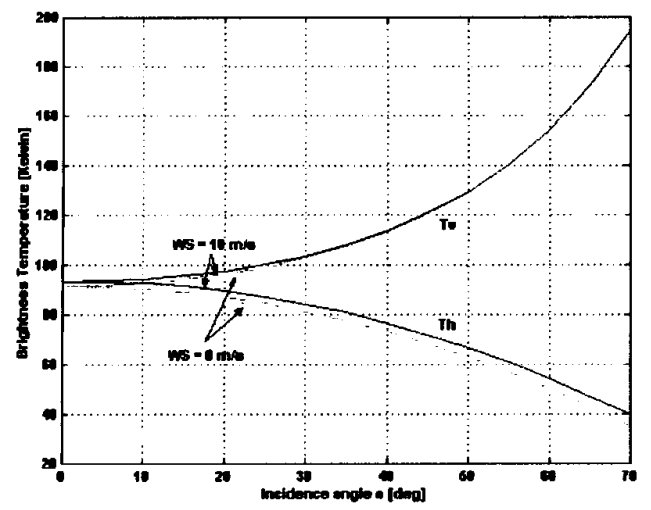

Figure 3. Simulated vertical and horizontal brightness temperatures for wind speed 0 and $10 \mathrm{~m} / \mathrm{s} @ 15^{\circ} \mathrm{C} \& 36 \mathrm{psu}$.

Further, the scanning configuration of SMOS presents new challenges:

- Two-dimensional imaging of the scene, with varying incidence angles and pixel resolution as the pixel travels trough the alias-free field of view;

- Not yet well understood azimuthal dependence of the first two Stokes parameters (Tv, Th) with wind direction;

- Unknown signature of the $3^{\text {rd }}$ and $4^{\text {th }}$ Stokes parameters $(\mathrm{U}, \mathrm{V})$ and their azimuth/elevation dependence with wind speed;

- Effect of sea foam at L-band;

- Polarization mixing between vertical and horizontal polarizations due to the relative orientation between the antenna frame and the pixel's local referece frame;

- Feasibility of accurate retrieval of $U$, and eventually $V$, assuming that Faraday rotation due to the ionospheric effects has been corrected for by other means.

A Wind and Salinity Experiment (WISE) is being prepared to answer the first four challenges above. The campaign will take place at Casablanca an oil platform (owned by Repsol) $40 \mathrm{~km}$ away from the Ebro river mouth. In this site high wind intensities of $90 \mathrm{~km} / \mathrm{h}(25 \mathrm{~m} / \mathrm{s})$ are not uncommon during October and November when the campaign is foreseen to take place (this year 2000).

The required measurements will be obtained from the following instrumentation:

- Two L-band radiometers: a fully polarimetric radiometer (Th, $T v, U$ and $V$ ) from the Polytechnic University of Catalonia at Barcelona (Spain), and a dual polarization radiometer (Th and $\mathrm{Tv}$ ) from the University of Massachusetts at Amherst (USA);
- Three oceanographic buoys from the ICM (Spain) and LODYC (France), that will measure sea surface salinity, sea surface temperature, wind speed and wind direction;

- Portable meteorological station for atmospheric pressure, temperature, relative humidity and rain rate;

- Stereo-camera from LODYC that will provide 3D images of the sea surface to measure sea surface rms slopes

- Video images of the antenna boresight from a video camera mounted on UPC radiometer,

- Infrared radiometer that will provide SST estimates.

The radiometers will be placed in the North side of the platform at about $32 \mathrm{~m}$ above the sea level, which will also avoid sun glint effects. The oceanographic buoys will be moored in the North side so as to collect ground truth data. Typical measurement sequences are:

- Scan in elevation (fixed azimuth angle), typically $\phi=0^{\circ}$ (North), starting at an incidence angle $\theta \mathrm{i}=20^{\circ}$, and ending at an incidence angle $\theta \mathrm{i}=65^{\circ}$, in angular steps of $5^{\circ}$ to $7.5^{\circ}$;

- Scan in azimuth at a fixed incidence angle at a particular value $\left(\theta \mathrm{i}=20^{\circ}-65^{\circ}\right)$, starting at an azimuth angle approximately $\phi=120^{\circ}$ West and ending at an azimuth angle approximately $\phi=75^{\circ}$ East in angular steps of $10^{\circ}$ to $15^{\circ}$.

The down-welling brightness temperature (atmospheric, cosmic and galactic noises) will be determined after each measurement by looking at 3 different elevation angles: nadir, horizon and zenith.

\section{CONCLUSIONS}

For many years it has been possible to measure sea surface temperature (SST) using infra-red satellite sensors. These measurements have provided a great deal of information about the physics of the oceans. Yet ocean circulation is dependent on water density - determined by both temperature and salinity. Measuring the salt content of the oceans is now becoming possible for the first time. This will lead to an improvement in our knowledge of the global ocean circulation.

\section{REFERENCES}

[1] Y. Kerr, J. Font, P. Wadteufel, A. Camps, J. Barà, I. Corbella, F. Torres, N. Duffo, M. Vall.llossera, G. Caudal, "Next Generation Radiometers: SMOS. A Dual Pol L-band 2D Apertures Synthesis Radiometers ", 2000 IEEE Aerospace Conference, March, 2000 Montana, USA.

[2] Camps, A., I. Corbella, J. Bará, F. Torres, "Radiometric Sensitivity Computation in Aperture Synthesis Interferometric Radiometry", IEEE Transactions on Geoscience and Remote Sensing, March 1998, GRS-36, No 2, pp 680-685. (Correction on GRS-36 No.5 Sept. 1998). 